\title{
VIDEO
}

\section{Double-balloon endoscopic management of iatrogenic perforation in the small bowel}
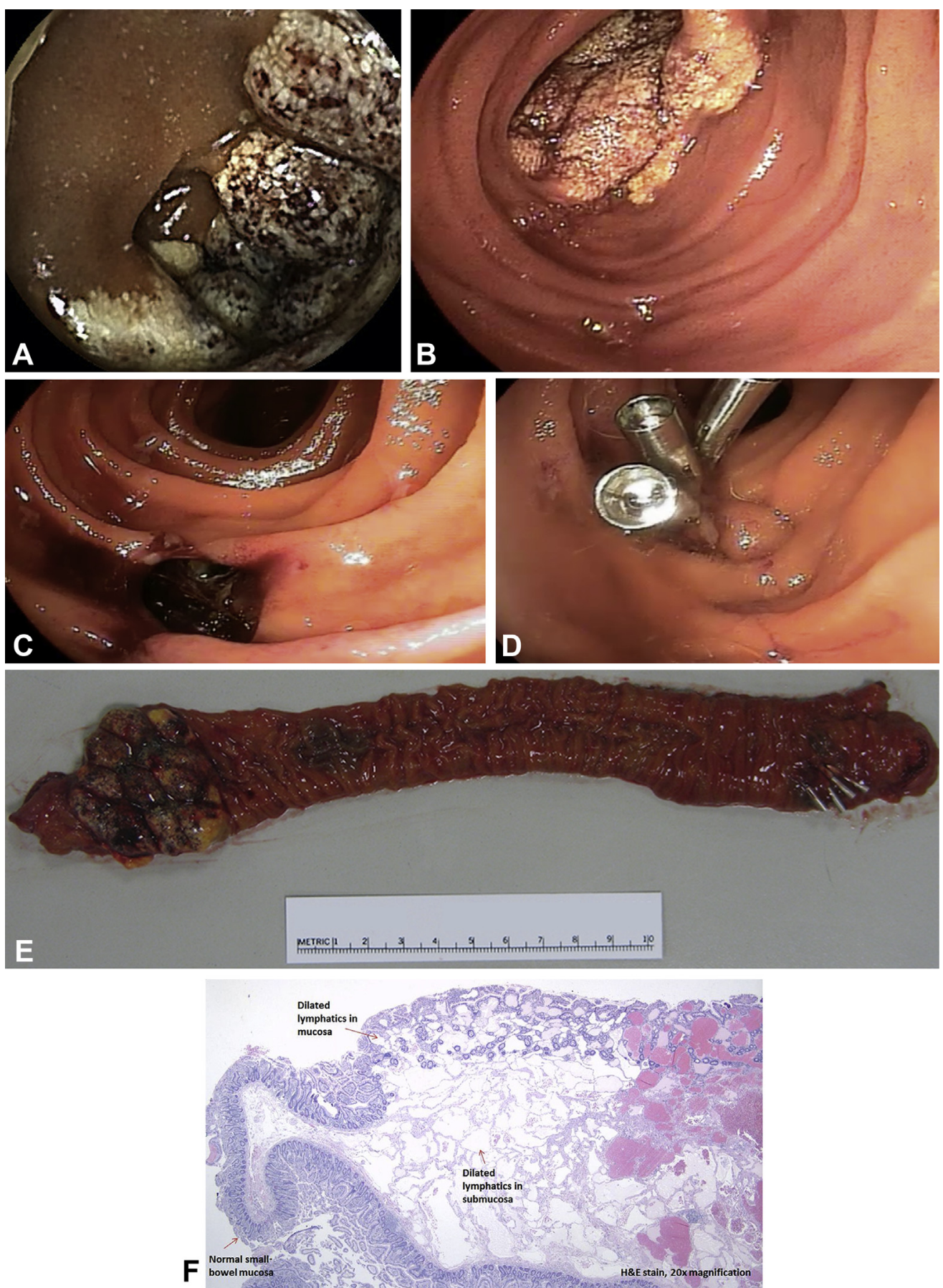

Figure 1. A, Video capsule endoscopic image of small-bowel lesion. B, Endoscopic image of small-bowel lesion. C, Endoscopic image of small-bowel perforation proximal to small-bowel lesion. D, Endoscopic image of successful closure of perforation with endoscopic clips. E, Gross specimen of small bowel with lesion (left) and closed perforation with endoscopic clips (right). F, Histology of lesion consistent with intestinal lymphangioma. 
This video report describes the case of a 48-year-old woman with rheumatoid arthritis and a history of nonsteroidal anti-inflammatory drug use with concomitant methotrexate immunosuppression, who presented with obscure GI bleeding and iron deficiency anemia. An upper endoscopy showed normal results. The result of a colonoscopy was essentially normal, with the presence of old blood in the right side of the colon. Video capsule endoscopy showed a polypoid mass lesion with dilatated lymphatics with a strawberry appearance and blood in the middle small bowel. Double-balloon enteroscopy was performed to identify, sample, and mark the mass lesion for subsequent internal laparoscopic small-bowel resection. After identification of the mass lesion, deep in the small bowel, the removal of a tissue sample with a standard biopsy forceps was complicated by an iatrogenic perforation, $1 \mathrm{~cm}$ in diameter, just proximal to the mass lesion, resulting from maximal insertion and torque of the enteroscope. Endoscopic hemoclips were used to close the defect. The patient underwent a same-day minilaparoscopy with resection of the mass lesion and closure of the perforation site (Video 1, available online at www.VideoGIE.org). There was no evidence of intestinal spillage at the time of surgery. The mass lesion was identified as a benign intestinal lymphangioma (Fig. 1). The patient did well postoperatively and was discharged home.
In conclusion, marking of intraluminal bleeding lesions deep in the small bowel allows easy identification of such lesions for internal laparoscopic resection. Torqueing of the enteroscope deep in the small bowel may create resistance to the passage of tools and increase the risk of perforation. Iatrogenic perforations deep in the small bowel can be successfully managed endoscopically with endoscopic hemoclips.

\section{DISCLOSURE}

All authors disclosed no financial relationships relevant to this publication.

Jonathan Cordova, DO, Irving Waxman, MD, Department of Gastroenterology, John Hart, MD, Department of Pathology, Carol Semrad, MD, Department of Gastroenterology, The University of Chicago Medicine, Chicago, Illinois, USA

Copyright (c) 2016 The Authors. Published by Elsevier, Inc. on behalf of the American Society for Gastrointestinal Endoscopy. This is an open access article under the CC BY-NC-ND license (http://creativecommons.org/ licenses/by-nc-nd/4.0/).

http://dx.doi.org/10.1016/j.vgie.2016.06.001 